Supporting information

\title{
Polymer surface transport is a combination of in-plane diffusion and desorption-mediated flights
}

Dapeng Wang, Huai-Ying Chin, Chunlin He, Mark P. Stoykovich, Daniel K. Schwartz*

Department of Chemical and Biological Engineering

University of Colorado, Boulder, CO 80309

*To whom correspondence should be addressed: daniel.schwartz@colorado.edu 
Materials - Homopolymer polystyrene (PS), poly(methyl methacrylate) (PMMA) and block copolymer polystyrene-block-poly(methyl methacrylate) (PS-b-PMMA) were purchased from Polymer Source, Inc. (Dorval, Quebec, Canada) and used as received. The number-average molecular weights of PS and PMMA are $51(P D I=1.05)$ and 50 $(P D I=1.09) \mathrm{kg} / \mathrm{mol}$, respectively. Three PS- $b$-PMMA copolymers are used to prepare PS-hexagonal arranged [PS- $b$-PMMA, $20: 50 \mathrm{~kg} / \mathrm{mol}, \mathrm{PDI}=1.07$ ], PMMA-continuous [PS- $b$-PMMA, $47: 53 \mathrm{~kg} / \mathrm{mol}, \mathrm{PDI}=1.12$ ] and PS-continuous [PS- $b$-PMMA, $53: 54$ $\mathrm{kg} / \mathrm{mol}, \mathrm{PDI}=1.16]$ surfaces. Alexa 647 labeled dextran with a molecular weight of 10 $\mathrm{kg} / \mathrm{mol}$ was purchased from Life Technologies.

Block copolymer thin film preparation - Fused silica wafers were immersed in a $70^{\circ} \mathrm{C}$ piranha solution for over $4 \mathrm{~h}$ followed by a UV-ozone treatment for $0.5 \mathrm{~h}$. The fusedsilica surface was neutralized by spin-coating a $0.15 \mathrm{wt} \%$ solution of random copolymer of PS-r-PMMA with a monomer ratio of 59:40:1 (PS:PMMA:Glycidyl Methacrylate) and annealing for $1 \mathrm{~h}$ at $190^{\circ} \mathrm{C}$. Random copolymer that was not cross-linked to the surface was removed by repeatedly spin-coating with toluene.

PS- $b$-PMMA thin films were prepared by spin-coating a $1.5 \mathrm{wt} \%$ block copolymer solution onto the previously prepared neutral substrates. To self-assemble the block copolymer thin film, thermal annealing was performed in an $\sim 2$ Torr vacuum at $190{ }^{\circ} \mathrm{C}$ for 3 days. 
Single-Molecule Tracking - Experiments were carried out by a Nikon TE-2000 microscope with a 60x water immersion objective in conjunction with a custom-built, prism-based illumination system. A CL-2000 diode pumped crystal laser with a wavelength of $641 \mathrm{~nm}$ was used as the excitation source for the fluorescent label. The laser was carefully oriented to produce an evanescent field at the interface. An EMCCD camera (model Cascade-II:512, Photometrics, Inc.) operated at $-95{ }^{\circ} \mathrm{C}$ was used to record movies of individual adsorbed dextran molecules. The fluorescence was collected by passing through a LP647 filter. For each surface, at least 100 movies with 100 s duration were continuously captured on different surface regions on multiple days. A custom-designed tracking algorithm was used to identify and track the fluorescent molecules. ${ }^{1}$ We removed totally immobile molecules by measuring the distance between the first and the last position; a threshold of $0.15 \mu \mathrm{m}$ was used to define immobilization.

CTRW Simulation on nanostructured surfaces - An improved 2D on-lattice CTRW simulation was designed to introduce randomly arranged PS domains in the following way. Each lattice site was equivalent to a $0.01 \times 0.01 \mu \mathrm{m}^{2}$ area, and an isolated PS domain was modeled as a square containing nine lattice sites. The isolated domains were randomly distributed on the lattice with an area fraction of $32 \%$. For comparison, the diffusion on a PS surface was studied as well. The walker, a dextran chain, was allowed to only occupy PS domains, initially chosen at random. Diffusion of molecules followed CTRW statistics with waiting periods alternating with flights. A given waitingtime was sampled from the distribution $\psi(\tau) \sim \tau^{-\alpha}$ with $\tau>t_{\mathrm{b}}$ where $t_{\mathrm{b}}$ was the lower limit. 
We empirically chose $\alpha=1.20$ and 1.18 on PS and PS-hexagonal surfaces to approximate the two-step decay of the waiting-time distribution in Figure 3b. Within a given waiting-time period, steps could either be totally immobile or exhibit in-plane diffusion within a contiguous PS region. A parameter $P$ controlled the ratio of these two types of waiting-time periods. The parameter $P$ was empirically determined by the relative fractions associated with the two peaks in Figure $3 \mathrm{c}$ in the main text. For the immobile waiting-time period, the position was determined by a Gaussian distributed noise function, $\exp \left(-r^{2} / I^{2}\right)$ with $I=0.035 \mu \mathrm{m}$ representing the experimental localization uncertainty. For waiting-time periods involving in-plane diffusion, the in-plane diffusion was designed to be Gaussian with $G(r, \Delta t)=\left(1 / \sqrt{4 \pi D_{2 D} \Delta t}\right) \cdot \exp \left(-r^{2} / 4 D_{2 D} \Delta t\right)$ where $D_{2 D}$ was the only adjustable parameter in the CTRW simulation. A random walk on the square lattice matrix with $n=r^{2} / 0.0001$ steps explores an area with a characteristic length scale of $r$. If a walker attempted to move to an adjacent non-PS lattice site, the move was not accepted and the walker remained in its original location. The position of the last step was further blurred by the Gaussian distributed noise function with $I=$ $0.035 \mu \mathrm{m}$. After waiting-time periods, a flight was executed as a two-dimensional onlattice random walk with a length drawn from the power law distribution $\mathrm{f}(r) \sim r^{-\beta}$ with $r_{\mathrm{b}}<$ $r<r_{\mathrm{e}}$ where $r_{\mathrm{b}}$ and $r_{\mathrm{e}}$ were the lower and upper limits of $r$. For desorption-mediated jumps, the distribution of step-size scales from $f(r) \sim r^{-1}$ to $f(r) \sim r^{-3}$ in different regimes. ${ }^{2}$ In the present work, we found that a value of $\beta=1.3$ was a good representation to sample $r$ over the relevant range of length scales. For each sampled $r$, the molecule executed a random walk on the square lattice with $n=r^{2} / 0.0001$ steps to explore the distance $r$, ignoring the nanostructures within the lattice matrix. Therefore, on the 
nanostructured surface, the last step may reside on a PMMA lattice site. In this case, the desorption-mediated step was executed again with a newly sampled $r$ until the flight ended on a PS lattice site.

The simulation time step was $0.1 \mathrm{~s}$. The distribution of trajectory simulation times was the same as the experimentally measured values and over $10^{5}$ trajectories were simulated for each system. The detailed simulation parameters are given as follows:

Table S1. Parameters used in Monte Carlo simulations for Figure 3 in the main text.

\begin{tabular}{|c|c|c|c|c|c|c|c|c|}
\hline No. & Type & $\alpha$ & $t_{b}(\mathrm{~s})$ & $\beta$ & $r_{b}(\mu \mathrm{m})$ & $r_{e}(\mu \mathrm{m})$ & $P$ & $D_{2 D}\left(\mu \mathrm{m}^{2} / \mathrm{s}\right)$ \\
\hline 1 & $\begin{array}{c}\text { Homogeneous } \\
\text { PS surface }\end{array}$ & 1.20 & 0.60 & 1.30 & 0.20 & 3.6 & 0.40 & 0.20 \\
\hline 2 & $\begin{array}{c}\text { PS-hexagonal } \\
\text { surface }\end{array}$ & 1.18 & 0.60 & 1.30 & 0.20 & 3.6 & 0.40 & 0.20 \\
\hline 3 & $\begin{array}{c}\text { Control } \\
\text { experiment on PS- } \\
\text { hexagonal surface }\end{array}$ & 1.20 & 0.60 & 1.30 & 0.20 & 3.6 & 0.40 & 0.20 \\
\hline
\end{tabular}




\section{Interpretation of the suppression of in-plane diffusion on the lamellar patterned surfaces}

It is interesting to consider the implications of the fact that the step-size distributions are similar, in the in-plane diffusion regime, on nanopatterned surfaces with different morphologies. For example, one might hypothesize that intermediate step sizes would be enhanced on lamellar patterned surfaces (particularly those with continuous PS domains) relative to the PS-hexagonal patterned surfaces if the diffusing molecules were guided along the continuous PS domains. The absence of such enhanced in-plane diffusion on lamellar surfaces suggests that the nanopattern not only acts as confinement for in-plane diffusion, but also exhibits an additional barrier effect. For example, this could be explained by a speculative scenario where diffusing molecules become stuck or inhibited when they encounter a boundary. 


\section{Figures cited in main text}

(a)

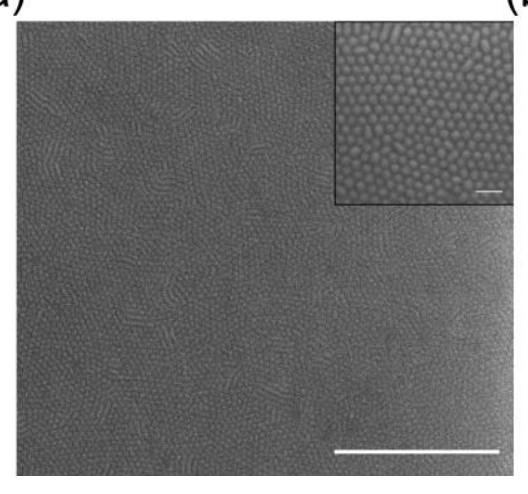

(b)

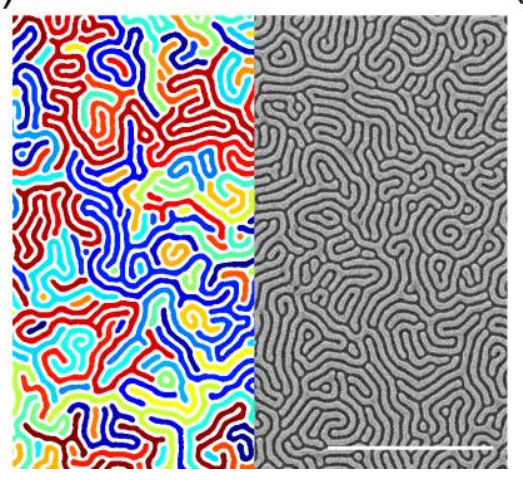

(c)

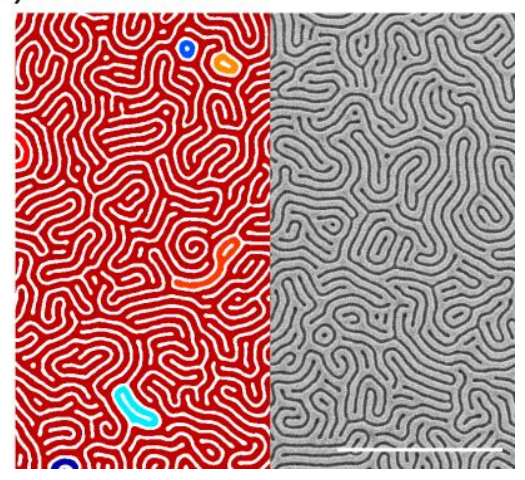

Figure S1. Representative SEM images of self-assembled PS-b-PMMA block copolymer thin films with different surface morphologies. (a) A film assembled from PS- $b$-PMMA with molecular weight of $20 \mathrm{~kg} / \mathrm{mol}$ (PS) and $50 \mathrm{~kg} / \mathrm{mol}$ (PMMA); The inset is the same as Figure 1a in the main text; (b) A PS- $b$-PMMA thin film with continuous PMMA domains prepared from block copolymer with a molecular weight of $47 \mathrm{~kg} / \mathrm{mol}$ (PS) and $58 \mathrm{~kg} / \mathrm{mol}$ (PMMA); the left side of the image shows the PS domains colorized based on their connectivity. Each distinctly colored PS domain denotes an isolated structure that is not connected to the other PS domains. The white regions represent the PMMA domains. Some colors are repeated due to a limited color palette. (c) A PS-b-PMMA thin film with continuous PS domains prepared from a block copolymer with a molecular weight of 53 $\mathrm{kg} / \mathrm{mol}$ (PS) and $54 \mathrm{~kg} / \mathrm{mol}$ (PMMA); the left side of the image shows the PS domains colorized based on their connectivity. The scale bar represents $1 \mu \mathrm{m}$. 


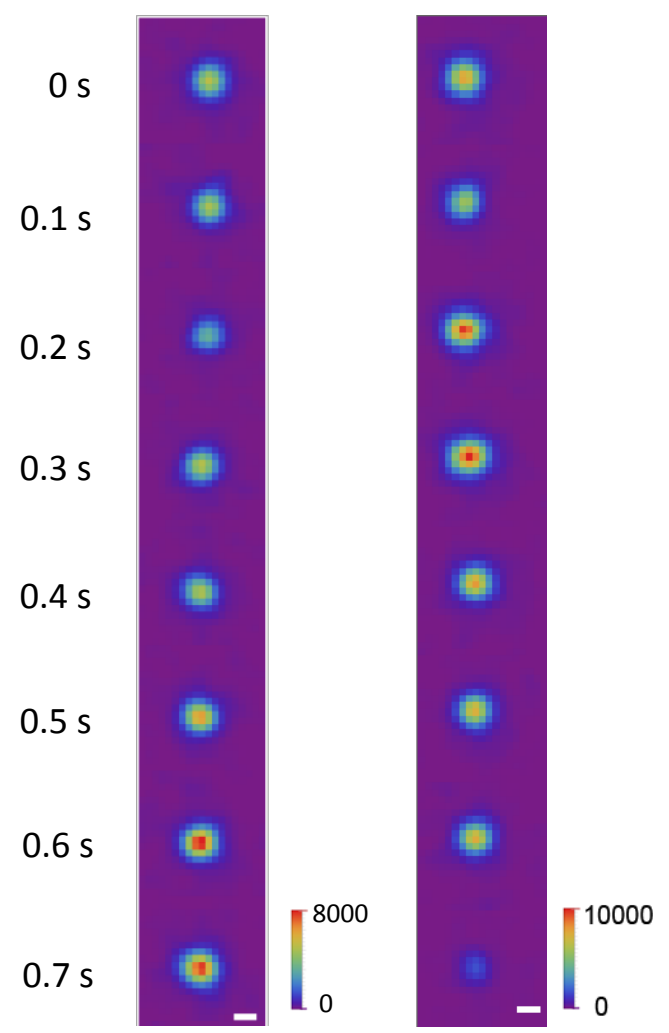

Figure S2. Representative fluorescence images of dextran molecules moving on a chemically homogeneous surface (pure PS) as time progresses. The scale bar represents $0.5 \mu \mathrm{m}$. The color bars on the right indicate the fluorescence intensity in arbitrary units. 


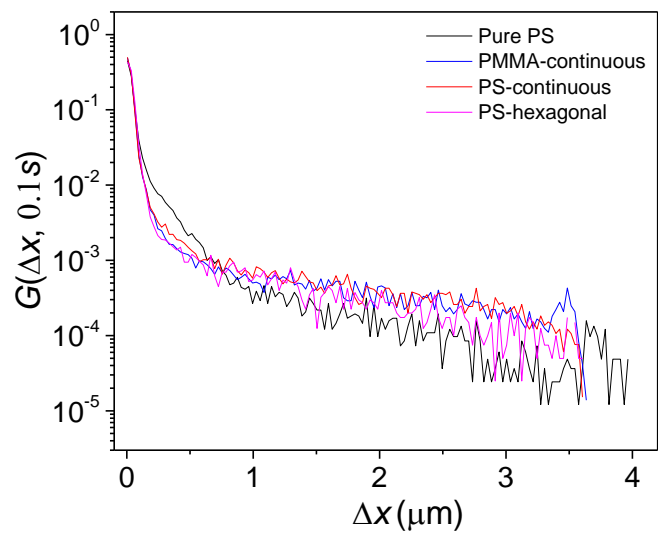

Figure S3. Step-size distributions $G(\Delta x, 0.1 s)$ of dextran on pure PS (black line), PS-hexagonal (pink line), and lamellar structures with continuous PMMA domains (blue line) or continuous PS domains (red line). 

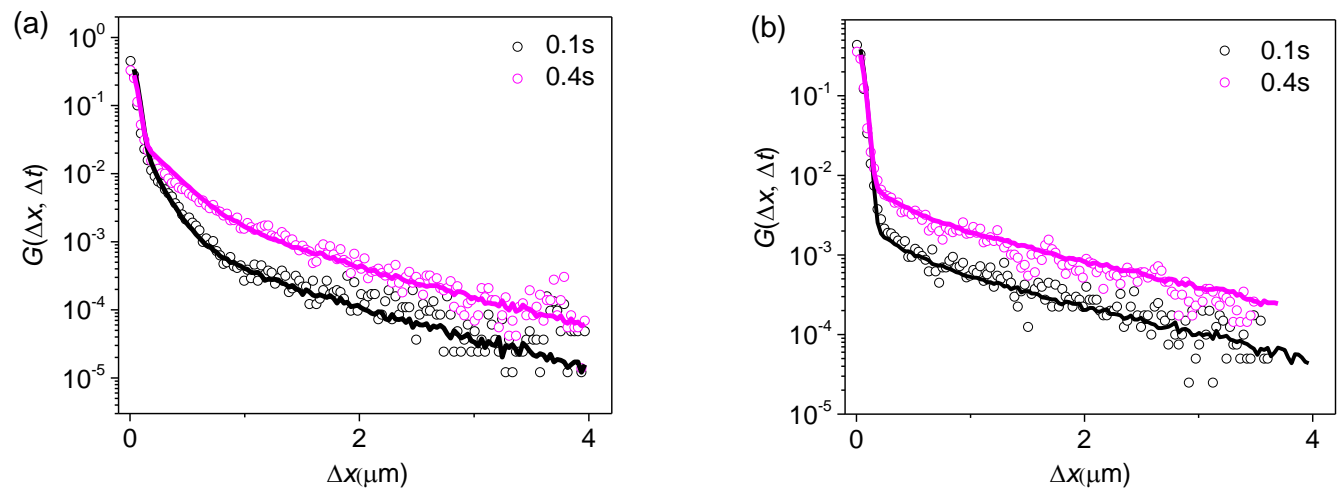

Figure S4. Step-size distribution of dextran on (a) homogeneous PS or (b) PS-hexagonal surfaces at $\Delta t=0.1,0.4 \mathrm{~s}$. The solid lines represent the simulation results at different lag times, using the parameter 1 (in Table S1) for (a) and 2 (in Table S1) for (b). 

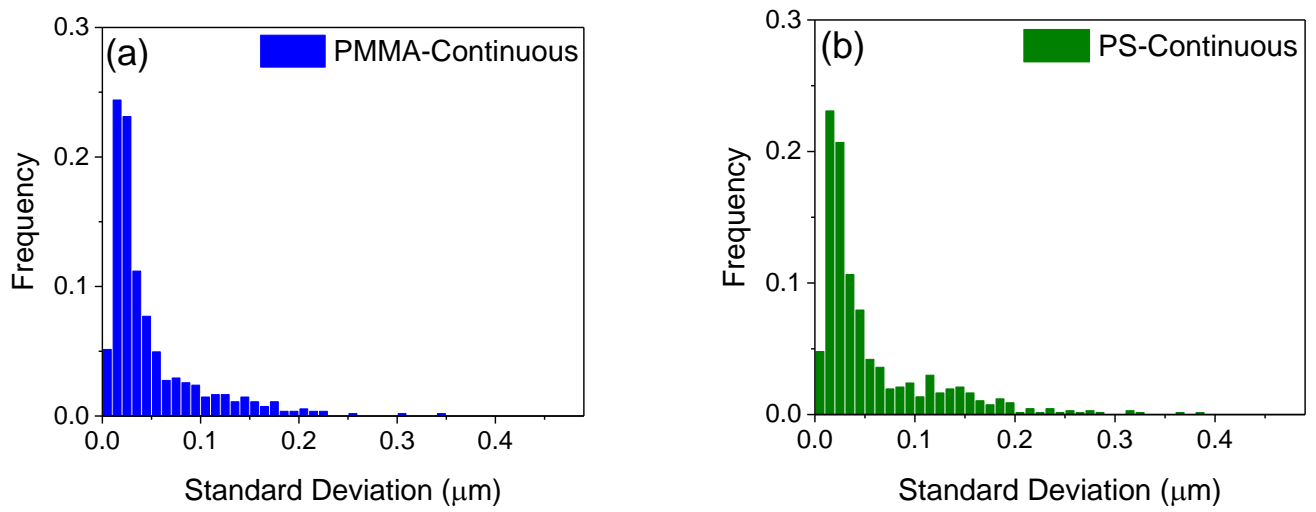

Figure S5. Distributions of standard deviation of position in each waiting-time period on (a) PMMA-Continuous and (b) PS-Continuous surfaces.

\section{References}

(1) Walder, R.; Kastantin, M.; Schwartz, D. K. Analyst 2012, 137, 2987-2996.

(2) Bychuk, O. V.; O'Shaughnessy, B. J. Phys. I/ 1994, 4, 1135-1156. 